\title{
Adler Synchronization of Spatial Laser Solitons Pinned by Defects
}

\author{
P. V. Paulau, ${ }^{1}$ C. McIntyre, ${ }^{2}$ Y. Noblet, ${ }^{2}$ N. Radwell, ${ }^{2}$ W. J. Firth, ${ }^{2}$ P. Colet,${ }^{3}$ T. Ackemann, ${ }^{2}$ and G.-L. Oppo ${ }^{2}$ \\ ${ }^{1}$ TU Berlin, Institut für Theoretische Physik, Hardenbergstr. 36, Sekr EW 7-1, 10623 Berlin, Germany \\ ${ }^{2}$ SUPA and Department of Physics, University of Strathclyde, 107 Rottenrow, Glasgow G4 ONG, United Kingdom \\ ${ }^{3}$ IFISC, (CSIC-UIB), Campus Universitat Illes Balears, E-07071 Palma de Mallorca, Spain
}

(Received 20 December 2011; published 23 May 2012)

\begin{abstract}
Defects due to growth fluctuations in broad-area semiconductor lasers induce pinning and frequency shifts of spatial laser solitons. The effects of defects on the interaction of two solitons are considered in lasers with frequency-selective feedback both theoretically and experimentally. We demonstrate frequency and phase synchronization of paired laser solitons as their detuning is varied. In both theory and experiment the locking behavior is well described by the Adler model for the synchronization of coupled oscillators.
\end{abstract}

DOI: 10.1103/PhysRevLett.108.213904

PACS numbers: 42.65.Tg, 05.45.Xt, 05.45.Yv

The formation of nonlinear structures such as patterns, solitons, oscillons, vortices, and spatio-temporal chaos, due to spontaneous breaking of translational symmetry in spatio-temporal systems, is nearly universal in science and nature [1]. Among such structures, dissipative solitons (self-localized states of driven, lossy systems) have attracted a lot of attention [2]. Laser cavity solitons (LCS) are optical dissipative solitons which possess both translational and phase invariance [2-4]. Temporal solitons in mode-locked lasers are a well-established type of LCS describable by the cubic-quintic complex GinzburgLandau (CGL) equation, which predicts that the interaction of LCS leads to phase-locked bound states with welldefined phases and separations [5]. Corresponding bound states have been observed experimentally in fiber lasers $[6,7]$. While similar behavior has been predicted for the spatial case in models of lasers with saturable absorbers $[3,8,9]$, experimental evidence has been lacking. Here we present experimental and theoretical evidence that the interaction of spatial LCS in real lasers is qualitatively different from that of temporal LCS, being governed by the archetypal Adler locking mechanism [10]. The Adler locking mechanism has relevance in biological clocks, chemical reactions, and mechanical and electrical oscillators [11]. In optics frequency locking of the Adler type was first observed in lasers with injected signals [12] with more recent generalizations to coupled lasers [13], the spatiotemporal domain [14], quantum dot lasers [15], and frequency without phase locking [16].

Early investigations on multiple spatial LCS in photorefractives [17] have been recently followed by their observation in semiconductor-based microresonators with either frequency-selective feedback [18,19] or saturable absorption [20,21]. Spatial LCS in real systems are usually pinned by defects resulting from fluctuations during the epitaxial growth process $[19,22]$. Besides fixing the position, these defects induce a shift in the LCS natural frequency. The diversity in natural frequencies among LCS pinned by defects is a critical ingredient of their description in real systems. We note that for temporal LCS, such as those arising in fiber lasers, the effects of longitudinal inhomogeneities are washed out by the propagation dynamics along the cavity axis, and every soliton sees the same material characteristics. Therefore, despite being suitable for temporal LCS, theoretical studies considering the interaction of identical LCS arising on a homogeneous background are not adequate to describe the dynamics of coupled spatial LCS.

We first present Adler frequency locking and phase synchronization of spatial LCS pinned by defects in a general CGL model with frequency-selective feedback where spatial variations of the cavity tuning parameter are used to simulate the presence of background defects. To show universality, defect induced Adler synchronization is then demonstrated in a model closer to the experimental realization where the saturable carrier dynamics are included [23]. Finally, the phenomenon is demonstrated experimentally in a vertical-cavity surface-emitting laser (VCSEL) with an external Bragg grating that provides frequency-selective feedback [19].

The interaction and locking phenomena which we observe in a semiconductor laser with feedback are well captured in a simple generic model consisting of a cubic CGL equation where solitons are stabilized by coupling to a linear filter equation [24]

$$
\begin{aligned}
& \partial_{t} E=g_{0} E+g_{2}|E|^{2} E-i \partial_{x}^{2} E+F+i n(x) E, \\
& \partial_{t} F=-\lambda F+\sigma E,
\end{aligned}
$$

where $E(x)$ is the intracavity field and $F(x)$ is the filtered feedback field. Note that the linear feedback equation breaks the Galilean invariance of the cubic CGL equation. We focus here on one transverse spatial dimension but similar results are expected in 2D. The time and space coordinates $(t, x)$ are scaled to $1 \mathrm{~ns}$ and $40 \mu \mathrm{m}$, respectively, so that $g_{0}$, describing linear gain and detuning, and $g_{2}$, describing nonlinear gain and dispersion, are dimensionless. We consider pure diffraction which is 
appropriate for VCSEL systems. The real function $n(x)$ describes spatial variations of the cavity tuning due to background defects that predominantly perturb the material refractive index. In the second part of Eq. (1) $\sigma$ is the feedback strength and $\lambda$ the filter bandwidth. The reference frequency is set to the filter peak. System (1) has exact solutions corresponding to stable single-frequency chirped-sech solitons [24]. Small variations of $n(x)$ lead to pinning and small changes in the soliton frequency.

We consider parameter values given by $g_{0}=-4+28 i$, $g_{2}=-96-48 i, \lambda=2.71, \sigma=162.6$, for which, in the ideal case with translational invariance, $n(x)=0$, system (1) has stable solitons with two free parameters: location and phase. The interaction of two such solitons makes them spiral slowly to fixed relative distances $L$ and phase differences around $\Phi=\phi_{2}-\phi_{1}=\pi / 2$ unless merging takes place. $\Phi$ equal to zero and $\pi$ are also possible but correspond to saddles that are either phase or distance unstable. Analytically the attainment of a bound state reduces to the analysis of two transcendental equations in the $(L, \Phi)$ phase space. The situation is very similar to that described in $[8,25]$ for bound solitons.

We now consider the interaction of solitons pinned by the potential $n(x)$ which is equal to zero everywhere except in the intervals $x_{j}-W<x<x_{j}+W$ where

$$
n(x)=\frac{-n_{j}}{2}\left[\cos \left(\frac{\pi\left(x-x_{j}\right)}{W}\right)+1\right]
$$

with $j=1,2$. The pinning potential is a smooth function of $x$ and the width $2 W$ of the defects is chosen to be close to the width at half-maximum of the LCS to help a quick convergence of the soliton distance to the final defect separation. Differences between the defects are described by the depths $n_{1}$ and $n_{2}$ of the pinning potential. The values of $n_{j}$ considered here preserve the structure of the LCS with only its frequency $\omega_{j}$ shifted.

If the defects are close enough in space, the soliton interaction locks their frequencies to a common value that depends on the average defect depth. The synchronization dynamics of the phase difference $\Phi$ between the pinned solitons relaxes to well-determined stationary values that depend on the defect detuning parameter $\Delta \omega=\omega_{2}-\omega_{1}$ generated by the choice of $n_{1}$ and $n_{2}$ values. The dependence of the stationary phase difference $\Phi$ on the detuning $\Delta \omega$ for numerical simulations of (1) is shown in Fig. 1 for $\left|x_{2}-x_{1}\right|=1.5$ space units. Locking and synchronization occur only in the range $|\Delta \omega|<\Delta \omega_{\text {th }}$. Very similar results have been obtained from numerical simulations of LCS in models of VCSELs with frequencyselective feedback that include the dynamics of the carriers and more realistic values of the linewidth enhancement factor [23] (see Fig. 1).

The archetypal equation for synchronization between two coupled oscillators is the Adler equation [10],

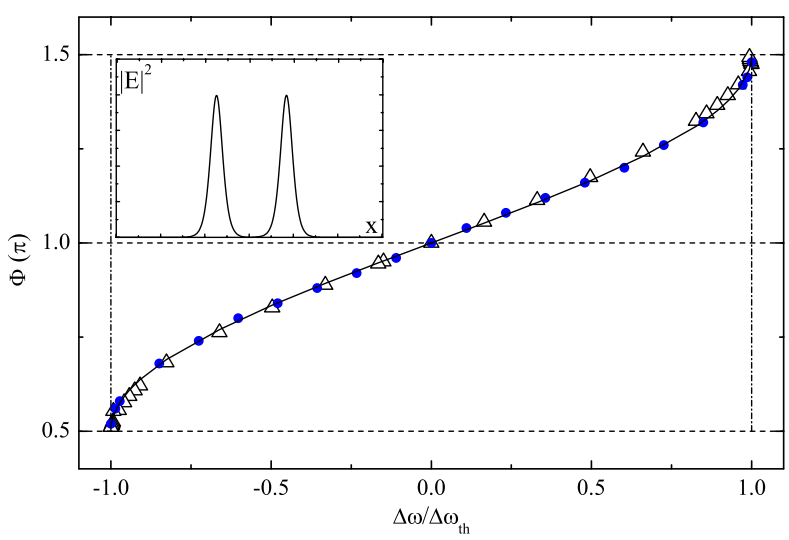

FIG. 1 (color online). Locked phase differences $\Phi$ of pinned LCS for different frequency detunings (controlled by the potential depths $n_{1}$ and $n_{2}$ ) from integration of Eq. (1) (dots, LCS separation of 5.3 soliton widths) and the model of Ref. [23] (triangles, LCS separation of 4 soliton widths). The solid line refers to the Adler equation (3). The inset shows the near-field profile of the intensity of two interacting LCS. Such a profile is almost constant across the Adler locking region.

$$
\frac{d \Phi}{d t}=\Delta \omega-\varepsilon \sin (\Phi)
$$

where in-phase and antiphase solutions are selected for zero detuning, $\Delta \omega=0$, depending on the sign of the coupling parameter $\varepsilon$ : for positive $\varepsilon$ the final stable state is $\Phi=0$; for negative $\varepsilon$ it is $\Phi=\pi$. A comparison of the results of the Adler equation with negative $\varepsilon$ and the simulations of the synchronization of LCS in both equations (1) and the model of Ref. [23] is presented in Fig. 1. The agreement is remarkable. Note that the $\pi / 2$ value observed in phase locking of dissipative solitons without defects [7] is now replaced by the $\pi$ value typical of Adler synchronization. In-phase and out-of-phase values have already been observed in numerical simulations of LCS in cubic-quintic CGL equations with regular variations of the background [26,27] although no Adler scenario is suggested. In particular, unless the period of the modulation is much larger than the length scales due to soliton interaction, the LCS are forced into different minima of the potential and do not experience any detuning difference any longer [27]. This is consistent with the $\pi$-phase states we observed for localized defects of equal depths $\left(\left|n_{2}-n_{1}\right|=0\right)$.

To characterize the Adler locking both in the spatial and temporal domains, we display the time averaged far field images in the top part of Fig. 2 and the optical spectra in the bottom part of Fig. 2 for two points inside $\left(\Delta \omega / \Delta \omega_{\text {th }}=0\right.$ and 0.99) and one outside the Adler region $\left(\Delta \omega / \Delta \omega_{\text {th }}=\right.$ $2)$, respectively. Progressive change of the LCS phase difference $\Phi$ [from $\pi$ in Fig. 2(a) to around $1.5 \pi$ in Fig. 2(b)] is reflected in the change in the symmetry of the fringe pattern. Far field fringes are well defined in the region where the LCS are locked in frequency [see the full overlap of the soliton peaks in the frequency spectrum in 

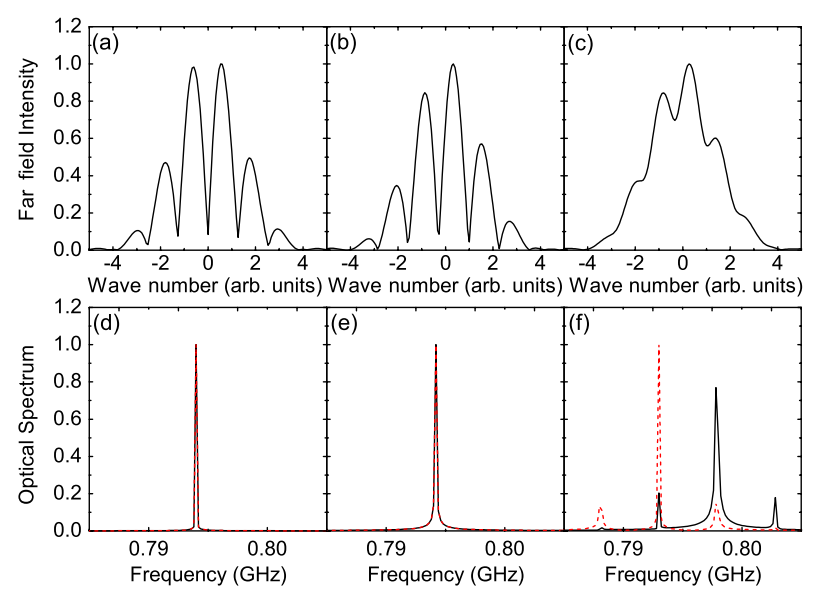

FIG. 2 (color online). Far field fringes (a)-(c) averaged over $2 \mu \mathrm{s}$, and optical spectra (d)-(f) for a time window of $5 \mu \mathrm{s}$, for $\Delta \omega / \Delta \omega_{\text {th }}=0(\mathrm{a}, \mathrm{d}), 0.99(\mathrm{~b}, \mathrm{e})$, and 2.0 (c,f) obtained from simulations of the model of Ref. [23]. In (d,e) the LCS spectral peaks (dashed and solid lines) overlap.

Figs. 2(d) and 2(e)] indicating a strong interaction. For detunings much larger than the locking range, the fringe visibility disappears and the spectrum is formed just by the lines of the individual solitons (not shown) corresponding to LCS operating independently. For detunings just outside the Adler locking region, however, some phase and spectral correlation survives due to nonuniform evolution of the relative phase [Figs. 2(c) and 2(f)].

The Adler locked state between LCS is a robust feature independent of initial conditions such as initial phases, frequencies, and sequential order of creation of the two LCS. Once the locked state is attained, one of the two LCS can be switched off by a short, localized perturbation to the carrier density at its location. Hence, LCS retain their solitonic properties in the phase-locked state in the sense that they are still individually bistable and optically controllable.

The experiment has been performed with a temperature tuned $981 \mathrm{~nm}$ VCSEL of $200 \mu \mathrm{m}$ circular aperture and a volume Bragg grating (VBG) with a single reflection peak at $981.1 \mathrm{~nm}$, a reflection bandwidth of $0.2 \mathrm{~nm}$ FWHM and a peak reflectivity of $99 \%$ [19]. The external cavity for the frequency-selective feedback is arranged in a self-imaging configuration that maintains the high Fresnel number of the VCSEL cavity and ensures local feedback compatible with self-localization (see Fig. 3). Small deviations from the selfimaging condition are not critical for the reported phenomena. The detection system comprises two chargecoupled-device cameras for near and far field imaging, and a scanning Fabry-Perot interferometer with a $10 \mathrm{GHz}$ free spectral range to measure the optical spectrum. When increasing the VCSEL injection current, several LCS appear at certain spatial locations favored by growth imperfections. On decreasing the current, each LCS displays hysteresis $[18,19]$. The experiment described below is performed at a bias current at which both LCS involved are

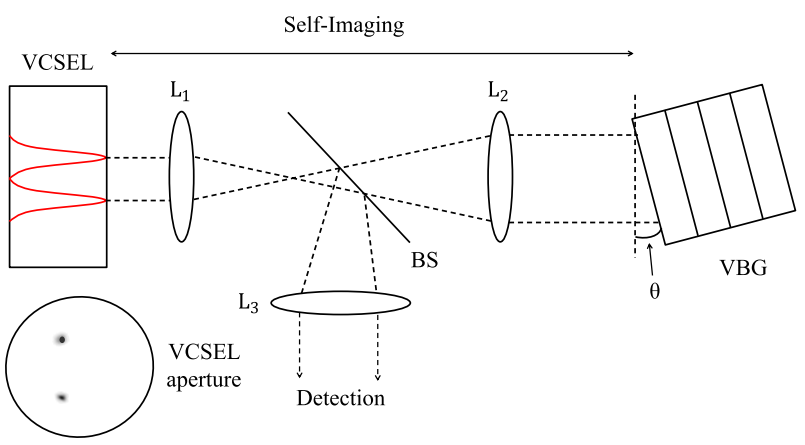

FIG. 3 (color online). Schematic diagram of a self-imaging cavity coupling a VCSEL to a frequency-selective element. The dotted lines correspond to the centers of the bundle of rays emitted by the soliton. The tilt angle $\Theta$ is exaggerated for clarity of display. The focal lengths of the intracavity lenses $L_{1}, L_{2}$ are $f_{1}=8 \mathrm{~mm}$ and $f_{2}=50 \mathrm{~mm}$, the total cavity length $L \approx 12 \mathrm{~cm}$ with a $1.23 \mathrm{GHz}$ free spectral range. $L_{3}$ images the near field of the VCSEL into the detection arm. The lower inset of the VCSEL aperture displays the near field of two interacting LCS.

individually bistable. Investigations were performed on pairs of different LCS with a distance of 30 to $80 \mu \mathrm{m}$. We focus here on a configuration of two LCS a distance of $79 \mu \mathrm{m}$, but the results are typical also for the other configurations. Each of these LCS is a coherent emitter but they are usually mutually incoherent due to the disorder [19,22].

Since it is experimentally awkward to vary the detuning between two LCS by locally changing the properties of the VCSEL itself, we use a piezo-electric transducer to minutely tilt the external cavity's end reflector (VBG) with respect to the optical axis. This leads to a differential change of the external cavity length for the two LCS and thus to a differential change in feedback phase, which can be incorporated into Eqs. (1) by making $\sigma$ complex. In this way, the frequency difference, i.e., the detuning $\Delta \omega$, between two LCS can be tuned [28]. During the scan, LCS position in near field and angular center in far field stay constant to better than 5\% and $2.5 \%$ of their width, respectively. When performing such a scan, a region of frequency and phase locking appears, identified in Fig. 4 by the region of high fringe visibility in the far field. These fringes are video integrated over a time of $20 \mathrm{~ms}$ (significantly longer than any intrinsic time scale) and last for seconds to hours depending on parameters. This illustrates that locking — once achieved by a careful alignment of the VBG-is a robust phenomenon.

As expected for the Adler scenario, in the locking region, the fringe phase varies smoothly and quasilinearly with the detuning of the external cavity. It is much more noisy outside where the visibility is low. The width of the locking range is close to the expected value of $\pi$. There is no significant phase hysteresis when the tilting is reversed [see the green (gray) solid and dashed lines in Fig. 4], again as expected for the Adler scenario. The transitions to and from frequency and phase locking are rather abrupt [Fig. 4, black curve]. For clarity, we show only a single sweep of 


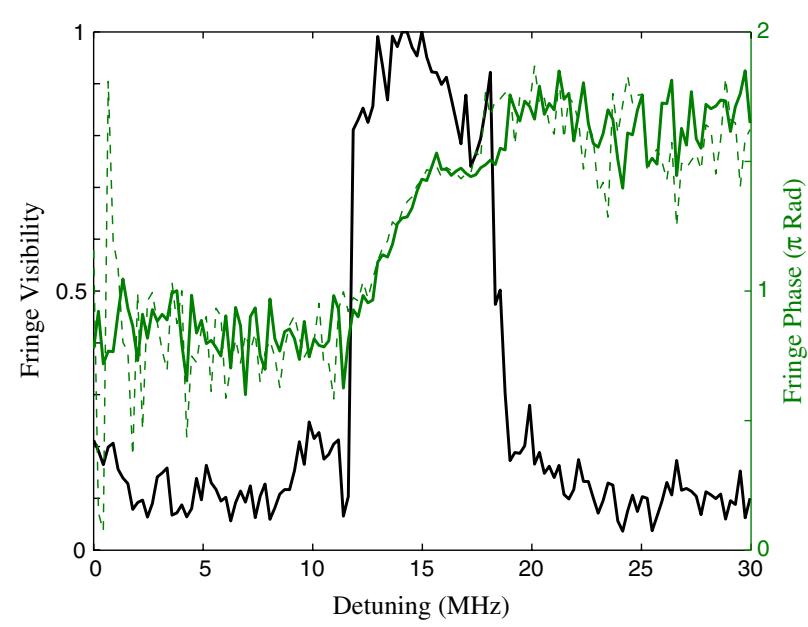

FIG. 4 (color online). Fringe visibility (black) and fringe phase (green curves, gray) as a function of the tilt angle that changes the difference between the feedback phases of the LCS. This difference is converted to a frequency scale by multiplying it by the free spectral range of the external cavity thus providing the change of the relative detuning between the two LCS in the external cavity. The zero of this detuning scale is arbitrary. The solid and dashed green (gray) curves are obtained for scanning the tilt back and forth. The fringe phase is obtained from the phase of a cosine-wave fitted to far field profiles like those in the upper row of Fig. 5. Other parameters: Temperature $69^{\circ} \mathrm{C}$, current $I=373 \mathrm{~mA}$.

the fringe visibility, because there is significant jitter at the transition points.

Figure 5 shows experimental far field fringes (upper part) and the corresponding optical power spectra (lower part), to be compared with the numerical results of Fig. 2. When the fringe visibility is high [Figs. 5(a) and 5(b)], the two LCS have the same frequency [Figs. 5(d) and 5(e)]. Weak side modes indicate residual excitation of neighboring external cavity modes. The change in fringe phase from $\pi$ [Fig. 5(a)] to $1.5 \pi$ [Fig. 5(b)] is reflected in the change in symmetry of the fringe pattern. Outside the locking region fringe visibility becomes very small [Fig. 5(c)] and the two LCS operate mainly on different external cavity modes.

A residual fringe visibility of $0.1-0.15$ in the nonlocked ranges of Fig. 4 indicates that some phase correlations survive even outside the strong locking regime. This can be attributed to a dynamical slowing down in the vicinity of the destroyed fixed point of the Adler equation but details of the transition scenario are likely to be influenced by features beyond the phase-only approximation of the Adler equation such as the multilongitudinal mode structure and possibly amplitude dynamics [29]. Longitudinal mode hopping of individual solitons can enable and/or quench the Adler dynamics thus explaining the jitter and limited locking range shown in Fig. 4. Within the locked region, however, the dynamics follow the Adler scenario with the locking phase being determined by the solitons differential feedback phase.
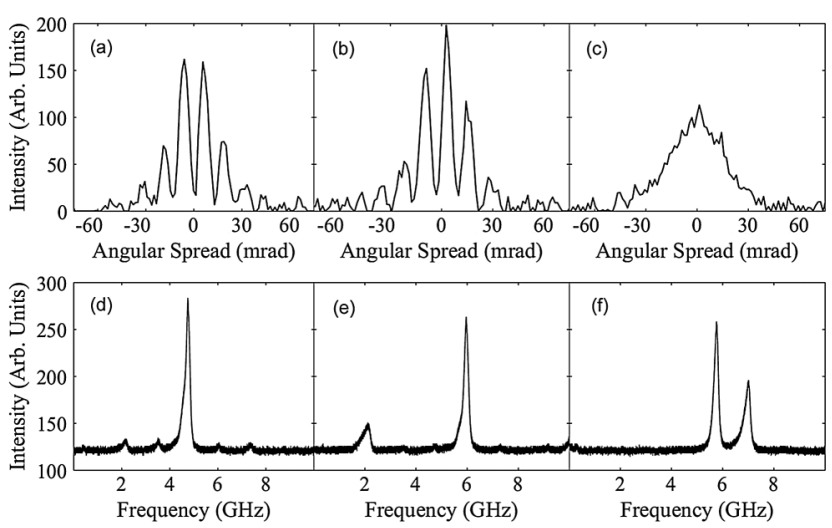

FIG. 5. Upper row: Cut through far field intensity distribution orthogonal to fringe orientation. Lower row: Optical power spectra. Left column (a,d) for detunings around $12 \mathrm{MHz}$, locked with a phase of $\pi$; center column (b,e) around $18 \mathrm{MHz}$, near the end of the locking region, locked with a phase of $1.5 \pi$; right column (c,f) around $22 \mathrm{MHz}$, unlocked, no clear fringes.

In conclusion, we have demonstrated spatio-temporal Adler synchronization without injection in semiconductor lasers with frequency-selective feedback. The synchronization is induced by spatial defects where the LCS are pinned and by changing the frequency of each LCS with respect to that of its neighbor. The presence of the defects breaks the translational symmetry, fixes the relative distance between solitons and locks the relative phase away from values close to $\pi / 2$ that are instead observed numerically in the absence of defects or experimentally in temporal-longitudinal systems. We expect similar considerations to apply to solitons in nonsemiconductor systems with phase symmetry such as photorefractive oscillators [17]. Synchronization behavior has been discussed in both continuous and coupled oscillator models [11]. Our study uses a continuous model, but synchronization is between "discrete" entities, the solitons. As such, we have provided a bridge between spatially extended media and coupled, predefined oscillators. Although we have demonstrated the validity of Adler's model for just two solitons, network synchronization in the spirit of Kuramoto's model (with coupling controlled by, e.g., the deviation from the selfimaging condition) should be possible with many LCS in a fruitful analogy with brain activity [30] and, possibly, with spatio-temporal excitability [14].

P. V.P. acknowledges support from SFB 910; C. M., Y. N., and N. R. from EPSRC DTA; P.C. from MICINN and Feder (FIS2007-60327, FISICOS and TEC200914101, DeCoDicA).

[1] M. Cross and H. Greenside, Pattern Formation and Dynamics in Nonequilibrium Systems (Cambridge University Press, Cambridge, 2009). 
[2] N. N. Akhmediev and A. Ankiewicz, Dissipative Solitons: From Optics to Biology and Medicine (Springer, Berlin, 2008).

[3] N. N. Rosanov, Spatial Hysteresis and Optical Patterns (Springer, Berlin, 2002).

[4] K. Staliunas and V. J. Sanchez-Morcillo, Transverse Patterns in Nonlinear Optical Resonators (Springer, Berlin, 2003).

[5] P. Grelu and N. Akhmediev, Nature Photon. 6, 84 (2012) and references therein.

[6] D. Y. Tang, W. S. Man, H. Y. Tam, and P. D. Drummond, Phys. Rev. A 64, 033814 (2001).

[7] Ph. Grelu, F. Belhache, F. Gutty, and J.-M. Soto-Crespo, Opt. Lett. 27, 966 (2002); Ph. Grelu, J. Beal, and J.-M Soto-Crespo, Opt. Express 11, 2238 (2003).

[8] A. G. Vladimirov, G. V. Khodova, and N. N. Rosanov, Phys. Rev. E 63, 056607 (2001).

[9] H. Vahed, R. Kheradmand, H. Tajalli, G. Tissoni, L. A. Lugiato, and F. Prati, Phys. Rev. A 84, 063814 (2011).

[10] R. Adler, Proc. IRE 34, 351 (1946); reprinted in Proc. IEEE 61, 1380 (1973).

[11] A. Pikovsky, M. Rosenblum, and J. Kurths, Synchronization. A Universal Concept in Nonlinear Sciences (Cambridge University Press, Cambridge, 2001).

[12] C. J. Buczek, R. J. Freiberg, and M. L. Skolnick, Proc. IEEE 61, 1411 (1973).

[13] L. Fabiny, P. Colet, R. Roy, and D. Lenstra, Phys. Rev. A 47, 4287 (1993).

[14] P. Coullet, D. Daboussy, and J. R. Tredicce, Phys. Rev. E 58, 5347 (1998).

[15] D. Goulding, S. P. Hegarty, O. Rasskazov, S. Melnik, M. Hartnett, G. Greene, J. G. McInerney, D. Rachinskii, and G. Huyet, Phys. Rev. Lett. 98, 153903 (2007).

[16] J. Thevenin, M. Romanelli, M. Vallet, M. Brunel, and T. Erneux, Phys. Rev. Lett. 107, 104101 (2011).
[17] G. Slekys, K. Staliunas, and C. O. Weiss, Opt. Commun. 149, 113 (1998).

[18] Y. Tanguy, T. Ackemann, W. J. Firth, and R. Jäger, Phys. Rev. Lett. 100, 013907 (2008).

[19] N. Radwell and T. Ackemann, IEEE J. Quantum Electron. 45, 1388 (2009); N. Radwell, C. McIntyre, A. J. Scroggie, G. L. Oppo, W. J. Firth, and T. Ackemann, Eur. Phys. J. D 59, 121 (2010).

[20] P. Genevet, S. Barland, M. Giudici, and J. R. Tredicce, Phys. Rev. Lett. 101, 123905 (2008).

[21] T. Elsass, K. Gauthron, G. Beaudoin, I. Sagnes, R. Kuszelewicz, and S. Barbay, Appl. Phys. B 98, 327 (2010).

[22] P. Genevet, M. Turconi, S. Barland, M. Giudici, and J. R. Tredicce, Eur. Phys. J. D 59, 109 (2010).

[23] A. J. Scroggie, W. J. Firth, and G.-L. Oppo, Phys. Rev. A 80, 013829 (2009).

[24] P. V. Paulau, D. Gomila, P. Colet, B. A. Malomed, and W. J. Firth, Phys. Rev. E 84, 036213 (2011).

[25] D. Turaev, A. G. Vladimirov, and S. Zelik, Phys. Rev. E 75, 045601(R) (2007).

[26] Y. He, B. A. Malomed, D. Mihalache, B. Liu, H. C. Huang, H. Yang, and H. Z. Wang, Opt. Lett. 34, 2976 (2009).

[27] W. Chang, N. Akhmediev, and S. Wabnitz, Phys. Rev. A 80, 013815 (2009); W. Chang, N. Akhmediev, S. Wabnitz, and M. Taki, J. Opt. Soc. Am. B 26, 2204 (2009).

[28] This follows the procedure used to control the detuning of coupled microchip lasers, e.g., Ref. [13], with additional coupled cavity effects.

[29] S. Wieczorek, B. Krauskopf, T. B. Simpson, and D. Lenstra, Phys. Rep. 416, 1 (2005).

[30] D. Cumin and C.P. Unsworth, Physica D (Amsterdam) 226, 181 (2007). 